\title{
Digitales Nachhaltigkeitsmanagement in Unternehmen
}

\author{
Ein serviceorientierter Ansatz zur Entwicklung einer Plattform für \\ datengestütztes Nachhaltigkeitsdatenmanagement
}

\begin{abstract}
Justus von Geibler, Julia Brandt, Wuppertal Institut für Klima, Umwelt, Energie, Lara Waltersmann, Robert Miehe, Fraunhofer-Institut für Produktionstechnik und Automatisierung IPA und Ralf Tesch,

\begin{abstract}
Die Stärkung der Digitalisierung im Nachhaltigkeitsmanagement und das Schaffen einer einheitlichen sowie konsistenten Datenbasis können Unternehmen maßgeblich unterstützen, steigenden Nachhaltigkeitsanforderungen zu entsprechen und die Transparenz zur Nachhaltigkeit des Wirtschaftens zu gewährleisten. Dieser Beitrag stellt Anforderungen und einen serviceorientierten Ansatz zur Entwicklung einer Plattform für datengestütztes Nachhaltigkeitsmanagement in produzierenden Unternehmen vor.
\end{abstract}

Die Transparenz zur Nachhaltigkeitsleistung von Unternehmen anhand nachvollziehbarer Nachhaltigkeitsdaten gewinnt zunehmend an Bedeutung, unter anderem durch den Druck von Politik, Behörden, Investoren und Gesellschaft sowie in Unternehmen gesetzten Zielen. Allerdings besteht häufig, insbesondere wegen fehlender IT-Unterstützung, kein einheitliches Management von Nachhaltigkeitsdaten. In diesem Beitrag wird auf konzeptionelle Grundlagen für die Entwicklung einer digitalen Plattform zum datengestützten Nachhaltigkeitsmanagement eingegangen und dazu folgende Aspekte adressiert: Bedeutung von Transparenz im Nachhaltigkeitsmanagement für Unternehmen, Ansätze zur Digitalisierung von Nachhaltigkeitsinformationen in Unternehmen sowie das Living-Lab-Konzept, das ermöglicht, nutzer- und serviceorientiert eine digitale Plattform für das datengestützte Nachhaltigkeitsmanagement aufzubauen. Diese Ansätze sollen im Rahmen eines Forschungsprojekts, das vom Bundesministerium für Bildung und Forschung gefördert wird, aufgegriffen werden.

Warum Transparenz im Nachhaltigkeitsmanagement?

Nachhaltiges Wirtschaften eröffnet neue
Geschäftsmöglichkeiten für Unternehmen und wird zunehmend zu einem entscheidenden Wettbewerbsfaktor [1]. Neben Politik und Behörden fordern auch Kunden und Investoren zunehmend eine Verbesserung der Nachhaltigkeitsleistung von Unternehmen anhand nachvollziehbarer Nachhaltigkeitsdaten [2]. Nachhaltigkeitsleistungen sollten zudem transparent und glaubhaft nachverfolgt werden können. Hierfür stehen im Bereich der Erfassung von Umweltauswirkungen diverse Methoden zur Verfügung, z. B. Lebenszyklusanalysen (LCA) oder Material Flow Cycle Analysis (MFCA). Allerdings stellen die Erhebung und das Management von hierfür benötigten Daten in Unternehmen aufgrund der Komplexität und Vielfalt der Datenquellen noch immer ein Problem dar; ein einheitliches Datenmanagement existiert kaum $[3,4]$.

Die nachvollziehbare Erfassung der Energie- und Ressourcennutzung und anderer Nachhaltigkeitsdaten ist eine Voraussetzung für die Verbesserung der Nachhaltigkeit in Unternehmen. Hier eröffnet die Digitalisierung vielversprechende Möglichkeiten bei der Datenerfassung [5], um betriebliche Energie- und Ressourcenverbräuche zu quantifizieren und Verbesserungspotenziale zu identifizieren $[4,6]$.
Digital Sustainability Management in Companies - A Service-Oriented Approach to Develop a Platform for Data-Driven Sustainability Management

The digitalization in sustainability management and the creation of a consistent database for sustainability data can significantly support companies in meeting increasing sustainability requirements and transparency regarding the sustainability performance. This paper presents a service-oriented approach for the development of a platform for data-driven sustainability management in manufacturing companies.

Keywords:

sustainability, sustainability management, data management, platform, digitalization

Dr. Justus von Geibler ist Co-Leiter des Forschungsbereichs Innovationslabore in der Abteilung Nachhaltiges Produzieren und Konsumieren am Wuppertal Institut für Klima, Umwelt, Energie.

Julia Brandt ist Senior Researcher im Forschungsbereich Innovationslabore der Abteilung Nachhaltiges Produzieren und Konsumieren am Wuppertal Institut für Klima, Umwelt, Energie.

Lara Waltersmann ist wissenschaftliche Mitarbeiterin in der Forschungsgruppe Nachhaltige Wertschöpfungssysteme am Fraunhofer-Institut für Produktionstechnik und Automatisierung IPA.

Dr. Robert Miehe ist Forschungsgruppenleiter für Nachhaltige Wertschöpfungssysteme am Fraunhofer IPA

Ralf Tesch ist Geschäftsführer der ConAct GmbH, Mainz. ConAct ist spezialisiert auf Lösungen für die Vernetzte Automatisierung in Industrieunternehmen

justus.geibler@wupperinst.org www.wupperinst.org 
Bild 1: Modell des Innovationsprozesses im Living Lab. Quelle: [23] in Anlehnung an Cooper (2002).

[1] Löser, F.: Strategic Information Systems Management for Environmental Sustainability: Enhancing Firm Competitiveness with Green IS. Berlin 2015

[2] Seidel, S.; Recker, J.; Vom Brocke, J.: Sensemaking and Sustainable Practicing: Functional Affordances of Information Systems in Geen of Information Systems in Green Transformations. In: MIS Quarterly 37 (2013) 4, S. 1275-1299.

[3] Schaltegger, S.; Burritt, R.: Contemporary environmental accounting. Issues, concepts and practice. Sheffield 2017

[4] Burritt, R.; Christ, K.: Industry 4.0 and environmental accounting: a new revolution? In: AJSSR 1 (2016) 1, S 23-38. DOl: 10.1186/s41180-016-

[5] Geibler, J. v:; Wiesen, K.; Mostyn, R.; Werner, M.; Riera, N. u. a.: Forming the Nucleus of a Novel Ecologica Accounting System. The myEcoCost Approach In: Key Engineering Materials, Vol 572 (2014), S.78-83.

VDI Zentrum Ressourceneffizienz $\mathrm{GmbH}$ (VDI ZRE): Ressourceneffiz enz durch Industrie 4.0 - Potenziale für KMU des verarbeitenden Gewerbes. VDI ZRE 2017. ULR: www.ressource-deutschland.de/fileadmin/ user_upload/downloads/studien/ VDI-ZRE Studie KI-betriebliche-Ressourceneffizienz Web bfpdf Abrufdam 15.09 _. Web

[7] Schaltegger, S.; Herzig, C.; Kleiber, O.; Klinke, T.; Müller, J.: Nachhaltig keitsmanagement in Unternehmen Von der Idee zur Praxis: Managementansätze zur Umsetzung von Corporate Social Responsibility und Corporate Sustainability. Lüneburg Corpora

[8] WBGU: Unsere gemeinsame digitale Zukunft. Zusammenfassung. 1 . Auflage. Berlin 2019.

[9] Miehe, R.; Waltersmann, L.; Sauer, A Bauernhansl, T. Sustainable production and the role of digital twinsBasic reflections and perspectives. J. Adv. Manuf. Process. 2021, 3.

[10] Barni, A.; Fontana, A.; Menato, S. Sorlini, M.; Canetta, L.: Exploiting the

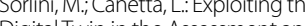
Digital Twin in the Assessment and Optimization of Sustainability Performances. In: International Conference on Intelligent Systems. 2018

[11] Waltersmann, L.; Kiemel, S.; Stuhlsatz J.; Sauer, A.; Miehe, R. Artificial Intelligence Applications for Increasing Resource Efficiency in Manufacturing Companies A Comprehenring Companies - A Comprehen-

[12] Conrad C.: ERP for ESG: Nachhaltig keitsmanagement mit Software-Systemen. In: CSR und Finance 2014. DOl: 10.1007/978-3-64254882-6_13.

[13] Bischof, $\bar{C}$.; Winkler, H.: Zeitgemäßes Umweltcontrolling mit SAP. In: Tschandl, M.; Posch, A. (eds): IntegTschand, M., Posch, A. (eds): Integ-
riertes Umweltcontrolling. Wiesbariertes Umw

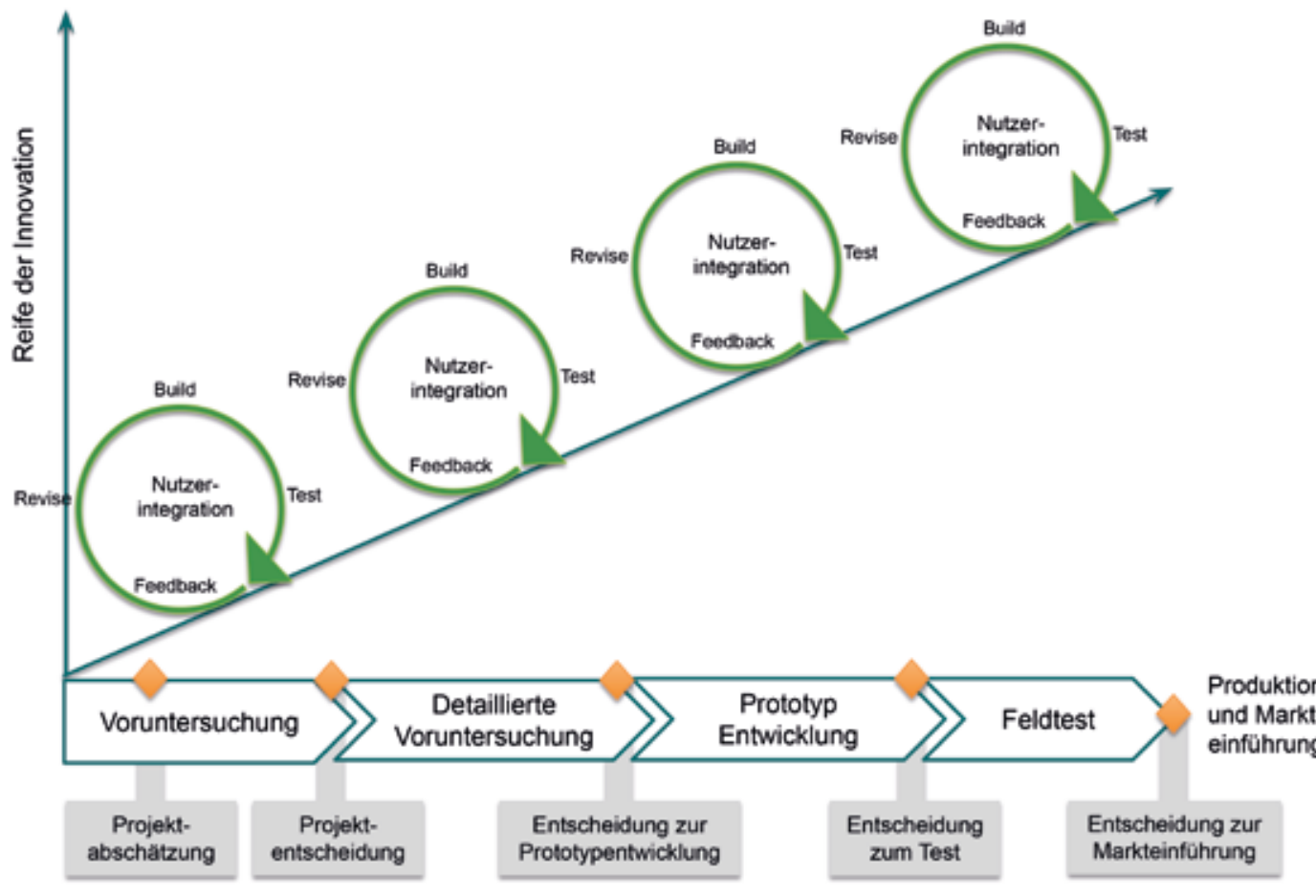

Digitale Ansätze für das

Nachhaltigkeitscontrolling

Das Nachhaltigkeitscontrolling mit seiner Informations-, Planungs-, Steuerungs- und Kontrollfunktion bildet die Grundlage für Transparenz und die Verbesserung der Nachhaltigkeitsleistung in Unternehmen. Die Informationserhebung und -verarbeitung von Nachhaltigkeitsdaten sind hierbei zwei der Kernaufgaben des Nachhaltigkeitscontrollings, das durch die steigenden Informationsbedarfe interner und externer Stakeholder zunehmend komplexer wird [7]. Digitale Ansätze können bei der Komplexitätsbeherrschung unterstützen. Die automatische Datenaufnahme und -verarbeitung über Sensoren und Algorithmen werden bereits von vielen Unternehmen umgesetzt. Nachhaltigkeit wird hierbei allerdings zumeist nicht berücksichtigt. Entsprechend betont der Wissenschaftliche Beirat der Bundesregierung Globale Umweltveränderungen (WGBU), dass Digitalisierung kein Selbstzweck ist, sondern zur Förderung der Großen Transformation zur Nachhaltigkeit aktiv eingesetzt und entsprechend gestaltet werden muss [8].

Für Unternehmen spielen insbesondere Nachhaltigkeitssoftware und der digitale Zwilling eine bedeutende Rolle [9, 10]. In Zukunft kann der vermehrte Einsatz von $\mathrm{KI}$-Anwendungen zu weiteren Nachhaltigkeitsverbesserungen führen $[6,11]$. Diverse Nachhaltigkeitssoftware bzw. betriebliche Umweltinformationssysteme unterstützen Unternehmen darin, Nachhaltigkeitsdaten für eingegrenzte Anwendungen, wie
Nachhaltigkeitsberichte oder Life Cycle Assessments, aufzunehmen und auszuwerten. Beispiele kommerzieller Software sind vielfältig [12]. Mit den weit verbreiteten Unternehmensdatenbanken, wie ERP-Systemen, ist zwar eine umfangreiche und strukturierte Datenbasis vorhanden, in der Teilaspekte der Nachhaltigkeit adressiert werden; dieses lässt jedoch keine Betrachtung aller relevanten Nachhaltigkeitsdaten zu und eine Anpassung der Datenstrukturen zur Aufnahme von weiteren Nachhaltigkeitsdaten ist mit einem hohen Aufwand verbunden [13].

Als Ausprägung eines digitalen Zwillings bietet die Verwaltungsschale eine gute Grundlage für die Datenerhebung und somit die Verwendung im Nachhaltigkeitscontrolling. Hier wurde in ersten Ansätzen gezeigt, dass diese zur Bewertung von Umweltauswirkungen herangezogen werden kann [14]. Die Lebenslaufakte nach DIN 77005 ist ebenfalls eine Ausprägung eines digitalen Zwillings und für eine bedarfsgerechte Informationsbereitstellung sowie Bewertung im Nachhaltigkeitsbereich generell geeignet [15]. Jedoch existieren weder für die Verwaltungsschale noch für die Lebenslaufakte Teilmodelle bzw. eine übergreifende Strukturierung der Nachhaltigkeitsdaten, sodass diese einheitlich für verschiedene Anwendungen im Kontext des Nachhaltigkeitscontrollings verwendet werden können.

Das vom Bundesministerium für Bildung und Forschung (BMBF) geförderte Projekt „Serviceorientierter Hub zur Verwertung von Nach- 
haltigkeitsinformation für produzierende Unternehmen (EcoHub)" schließt an diesen Forschungsbedarf im Bereich der Nachhaltigkeitssoftware als auch der Datenstrukturierung an. Es soll die Nachhaltigkeitsplattform „EcoHub“ für übergreifende Anwendungen entwickelt werden. Der Living-Lab-Ansatz bildet den zentralen Innovationsansatz bei der Entwicklung dieser Plattform.

\section{Der Living-Lab-Ansatz für die Entwicklung der EcoHub Datenplattform}

Im Kontext von Nachhaltigkeitsinnovationen und komplexen Entwicklungsumgebungen gewinnen nutzer- und anwendungsorientierte Innovationsprozesse, wie Ko-Kreation und Open-Innovation-Prozesse zunehmend an Bedeutung [16]. Die Öffnung von Innovationsprozessen sowie die gezielte Einbindung relevanter Anspruchsgruppen erhöhen die Chance, das Know-how einer breiteren Anzahl von Akteuren in Anspruch zu nehmen [17]. Gleichzeitig zeichnet sich ein Trend ab, bei dem sich Innovationsprozesse zunehmend an der Realwelt orientieren und praxisnahe Kontexte zur Entwicklung von Innovationen verwendet werden [18]. In diesem Zusammenhang gewinnt der LivingLab-Ansatz in der Innovationspraxis und der Nachhaltigkeitsforschung zunehmend an Bedeutung $[19,20]$.

Ein Living Lab ist ein offener Forschungs- und Innovationsansatz, mit dem Produkte und Dienstleistungen gemeinsam mit Nutzern und Stakeholdern entwickelt (Co-Creation) und praxisnah getestet werden können [21]. Dieser Ansatz zielt auf eine konsequente Praxis- und Nutzerorientierung in den zentralen Phasen der Entwicklung und Erprobung von technisch-gestützten Systemen ab [22]. Bei der Erprobung steht das iterative Lernen in einem strukturierten Prozess und experimentellen, praxisnahen Umfeld im Vordergrund (Bild 1).

Der Begriff des Labors wird im Living Lab über seine klassische natur- und ingenieurwissenschaftliche Bedeutung hinaus auf einen sozialen und ökologischen Kontext erweitert. Dafür öffnet sich der Innovationsprozess an zentralen Stellen, sodass neben den Entwicklern auch Anwender und weitere relevante Akteure im Anwendungsfeld einbezogen werden können [24]. Frühe Experimente und Tests in einer praxisnahen Umgebung helfen, Entwick- lungsfehler aufzudecken und wertvolle Feedbacks zu Akzeptanz und Nutzung zu erhalten $[25,26]$.

Der Entwicklungsansatz des Living Labs wird auch für die Entwicklung der EcoHub Datenplattform zum Nachhaltigkeitsmanagement genutzt. Dazu soll er iterativ und interaktiv gemeinsam mit Unternehmen und bezogen auf verschiedene Anwendungsfälle des Nachhaltigkeitsmanagements bezogen werden.

\section{Fazit und Ausblick}

Das digitale Management von Nachhaltigkeitsdaten bietet große Potenziale für das Nachhaltigkeitsmanagement in Unternehmen und eine Vielzahl von Anwendungen. Das vom BMBF geförderte Projekt „EcoHub“ ist mit der Zielsetzung gestartet, ein ganzheitliches Management von Nachhaltigkeitsdaten auf Basis einer einheitlichen Datenplattform in Unternehmen zu ermöglichen und Funktionen für das Nachhaltigkeitsmanagement in Form von flexiblen Services einfach nutzbar zu machen. Unter der Leitung vom Fraunhofer-Institut für Produktionstechnik und Automatisierung IPA wird mit zwei weiteren Forschungs- und Entwicklungspartnern, dem Wuppertal Institut und der ConAct $\mathrm{GmbH}$, sowie 13 Praxispartnern aus der Wirtschaft hierzu eine prototypische, nutzerzentrierte Datenarchitektur entwickelt und am Beispiel einer Plattform umgesetzt, dem serviceorientierten Hub zur Verwertung von Nachhaltigkeitsinformation für produzierende Unternehmen (EcoHub). Die Entwicklung der Eco-Hub Plattform (insb. die Identifikation von Anforderungen, die prototypische Umsetzung und die Validierung) erfolgt auf Basis des nutzerintegrierenden Living-Lab-Ansatzes anhand von neun Use-Cases, in welchen in Kooperation mit produzierenden Unternehmen gleichzeitig konkrete Verbesserungen in Bezug auf Nachhaltigkeit umgesetzt und quantifiziert werden.

Dieser Beitrag entstand im Rahmen des Projekts "Serviceorientierter Hub zur Verwertung von Nachhaltigkeitsinformation für produzierende Unternehmen", das vom Bundesministerium für Bildung und Forschung im Rahmen der Förderinitiative "Sozial-ökologische Forschung" unter dem Kennzeichen 02J20E528 gefördert wird.

Schlüsselwörter:

Nachhaltigkeit, Nachhaltigkeitsmanagement, Datenmanagement, Plattform, Digitalisierung
[14] Al Assadi, A.; Waltersmann, L.; Miehe, R.; Fechter, M.; Sauer, A.: Automated Environmental Impact Assessment (ElA) via Asset Administration Shell. In:Weißra In:Weißgraeber, P., Heieck, F.; Ackermann, C. (eds). Advances in Automotive Production Technology Theory and Application. ARENA2036. Berlin Heidelberg 2021 https://doi.org/10.1007/978-3-66262962-8_6.

[15] DIN und DKE: Deutsche Normungsroadmap Industrie 4.0 Version 3. 2018.

[16] Warnke, P.; Koschatzky, K.; Dönitz, E.; Zenker, A. u. a.: Opening up the innovation system framework towards new actors and institutions. Discussion Papers Innovation Systems and Policy Analysis, No. 49. Karlsruhe 2016.

[17] Bartle, M.: Open Innovation. Der offene Umgang mit Wissen verändert das Innovationsmanagement. dert das Innovationsmanagement.
In: pen Journal of Knowledge Management 1 (2010).

[18] Schneidewind, U.; Singer-Brodowski, M.:Vom experimentellen Lernen zum transformativen Experimentieren. Zeitschrift für Wirtschaftsund Unternehmensethik, 16 (2015) 1, S. 10-23.

[19] Schäpke, N. Stelzer, F.; Caniglia, G.; Bergmann, M.; Wanner, M. u. a.: Jointly experimenting for transformation? Shaping real-world laboratories by comparing them. GAIA-Ecological Perspectives for Science and Society 27 (2018) 1, S. 85-96.

[20] Ley, B:; Ogonowski, C: Mu, M.; Hess, J.; Race, N. u. a.: At home with users: a comparative view of Living Labs. Interacting with Computers, 27 (2015) 1, S. 21-35.

[21] Geibler, J.v.; Erdmann, L.; Liedtke, C.; Rohn, H.; Stabe, M. u. a.: Living Labs für nachhaltige Entwicklung. Wuppertal Spezial Nr. 47. Wuppertal 2013.

[22] Baedeker, C:Piwowar, J:Themann, P.; Grinewitschus, V.; Krisemendt, B. u. a.: Interactive Design to Encourage Energy Efficiency in Offices: Developing and testing a User-Centred Building Management System based on a Living Lab Approach. In: Sustainability, Volume 12 (2020) 17, 6956.

[23] Geibler, J.v:Echternacht, L:Stadler, K.; Liedtke, C.; Hasselkuß, M. u. a.: Nachhaltigkeitsanforderungen und -bewertung in Living Labs: Konzeption eines Bewertungsmodells. Wuppertal 2016

[24] Schuurman, D.; Herregodts, A.-L.; Georges, A.; Rits, O.: Innovation Management in Living Lab Projects: The Innovatrix Framework. Technology Innovation Management Relogy Innovation Managem
view 9 (2019) 3, S. 63-73.

[25] Liedtke, C., Baedecker, C., Hasselkuß, M., Rohn, H., Grinewitschus, V.: User-Integrated Innovation in Sustainable LivingLabs: An Experimental Infrastructure for Researching and Developing Sustainable Ching and Developing Sustainable Product Service Systems. Journal of Cleaner Production, 97. (2015), 106-116.

[26] Geibler, J. v.; Piwowar, J.; Greven, A.: The SDG-Check: Guiding Open Innovation towards Sustainable Development Goals. Technology Innovation Management Review, 9 (2019) 3, S. 20-37. 